\title{
Preclinical imaging of kallikrein-related peptidase 2 (hK2) in prostate cancer with a ${ }^{111}$ In-radiolabelled monoclonal antibody, 11B6
}

Oskar Vilhelmsson Timmermand ${ }^{1 *}$, David Ulmert ${ }^{2}$, Susan Evans-Axelsson ${ }^{3}$, Kim Pettersson ${ }^{4}$, Anders Bjartell ${ }^{3}$, Hans Lilja $2,5,6,7$, Sven-Erik Strand ${ }^{1,8}$ and Thuy A $\operatorname{Tran}^{8}$

\begin{abstract}
Background: Prostate cancer is a leading cause of death in the male population of the western world. Human kallikrein-related peptidase 2 (hK2) is abundantly expressed in malignant prostatic tissue, and its gene, KLK2, is regulated by the androgen receptor. 11B6 is a murine lgG monoclonal antibody directed against free human hK2. In this study, we performed a preclinical evaluation of ${ }^{111}$ In-labelled $11 \mathrm{~B} 6$ in mouse xenografts to investigate its potential in the clinical staging and assessment of metastatic prostate cancer.

Methods: $11 \mathrm{~B} 6$ was radiolabelled with ${ }^{111}$ In through CHX-A"-DTPA chelation. In vivo biodistribution and uptake of ${ }^{111}$ In-DTPA-11B6 were measured until $168 \mathrm{~h}$ post-injection in NMRI nude mice bearing subcutaneous LNCaP xenografts. The binding specificity to hK2 was evaluated by both in vivo competitive binding assays with excess non-labelled 11B6 and hK2-negative DU145 xenografts. SPECT/CT imaging of subcutaneous and intra-tibial LNCaP xenografts was used to visualize the tumours.

Results: Tumour uptake of ${ }^{111}$ In-DTPA-11B6 in LNCaP xenografts was 19\% $\pm 0.78 \% \mathrm{IA} / \mathrm{g}$ at $48 \mathrm{~h}$, giving a tumour-toblood ratio of 1.6, which increases to 2.4 at 1 week post-injection. Accumulation was low in other organs except for the salivary glands, which is probably the result of cross-reactivity with mouse kallikreins. Significantly lower tumour accumulation was observed in competitive assays and DU145 xenografts. SPECT/CT imaging could clearly visualize the subcutaneous and intra-tibial LNCaP xenografts.
\end{abstract}

Conclusions: Our study demonstrates the potential of ${ }^{111}$ In-DTPA-11B6 for the detection of metastatic prostate cancer and monitoring anti-androgen therapy, as it exhibits an increased uptake and accumulation in viable tumour when compared to normal tissue. A humanised version of the 11B6 monoclonal antibody is currently under evaluation.

Keywords: Prostate cancer; Human kallikrein-related peptidase 2; Human kallikrein gene family; ${ }^{111}$ In-DTPA-11B6; Molecular imaging

\section{Background}

Although improvements have been made in approaches for both diagnosis and treatment, prostate cancer (PCa) remains the second leading cause of cancer-related deaths in the male population of the western world [1]. The ratio of local-regional $\mathrm{PCa}$ to that of metastatic disease has increased since the introduction of prostate-specific antigen (PSA) testing, subsequently raising questions regarding

\footnotetext{
* Correspondence: oskar.vilhelmsson_timmermand@med.lu.se

${ }^{1}$ Department of Medical Radiation Physics, Lund University, Barngatan 2:1,

S-211 85 Lund, Sweden

Full list of author information is available at the end of the article
}

overdiagnosis $[2,3]$. Nevertheless, there is a need for new molecular imaging moieties to improve the clinical staging and monitoring of $\mathrm{PCa}$ in order to identify the optimal course of treatment for the disease.

Today, molecular imaging methods based on metabolic markers such as ${ }^{18}$ F-FDG (fluorodeoxyglucose) and ${ }^{18} \mathrm{~F} /{ }^{11} \mathrm{C}$-Choline are commonly used; however, there are known limitations for these tracers. ${ }^{18} \mathrm{~F}$-FDG works well in a more advanced disseminated disease, but is ineffective in detecting localized PCa because of its lower glycolytic uptake compared to other neoplasms [4]. Also, the normal elimination of ${ }^{18}$ F-FDG through the urinary bladder 
can mask the uptake in the prostate and regional lymph nodes [5]. ${ }^{18} \mathrm{~F} /{ }^{11} \mathrm{C}$-Choline is the most common radiotracer in $\mathrm{PCa}$ imaging today, and while they perform better than ${ }^{18}$ F-FDG, they are still limited in their ability to differentiate between a localized $\mathrm{PCa}$ and benign disease [6].

Molecular imaging of androgen receptor (AR) signalling holds the promise for more accurate disease evaluation and therapeutic monitoring, with additional implications for hormonal treatment and radiation therapy in PCa. PCa growth is dependent on androgens that signal through the AR and plays a fundamental role in cancer cell proliferation, apoptosis and invasion/metastasis [7]. A newly developed radiotracer, ${ }^{18} \mathrm{~F}$-FDHT $\left(16 \beta-\left[{ }^{18} \mathrm{~F}\right]\right.$ fluoro-5 $\alpha$-dihydrotestosterone), enables the imaging of AR expression. Furthermore, preliminary clinical studies demonstrated that ${ }^{18} \mathrm{~F}$-FDHT might provide for the imaging of $\mathrm{AR}$ expression during disease progression in castration-resistant $\mathrm{PCa}$ [8].

The commercially available ProstaScint ${ }^{\oplus},{ }^{111}$ In-capromab pendetide, targets an intracellular epitope of the prostate-specific membrane antigen (PSMA) [9], another surrogate of AR signalling. Promising results were recently reported using a new antibody (J591) against an extracellular domain of the PSMA molecule and presents as a possibility to suppress PSMA expression following antiandrogen treatments [10]. However, the PSMA expression in other tissues such as the human brain [11] hampers this approach to PCa imaging.

An alternative strategy could be to target AR-dependent antigens such as PSA and hK2 as they express almost exclusively in the prostate tissue $[12,13]$. Both PSA and $\mathrm{hK} 2$ are serine proteases, encoded by the human kallikrein genes $K L K 3$ and $K L K 2$ located on chromosome 19, respectively, and are well characterized as AR-regulated genes [14-16]. These kallikreins are produced by the same secretory luminal cells in the prostate and share an $80 \%$ amino acid homology, as well as several structural similarities [15-17]. Importantly, the PSA and hK2 antigens are abundantly expressed in malignant prostate tissue throughout all clinical stages. Recent publications on the 5A10 [18] and PSA30 antibodies [19] have explored the concept of PSA imaging, including targeting the free, unbound forms of prostate-specific antigen (fPSA). Results from animal studies using the PSA30 antibody showed a selective uptake in LNCaP tumours in vivo; however, the retention was faint and most likely due to shortcomings in the ${ }^{125}$ I-labelling method. In addition, ${ }^{89} \mathrm{Zr}$-labelled 5A10 exhibited better imaging capabilities but was accompanied by increased liver uptake. Nonetheless, this PET tracer was sufficient in detecting AR-dependent changes in PSA expression levels in mouse tumour lesions, as well as in distinguishing PCa cells within bone lesions both of which may be useful in the staging and clinical evaluation of advanced prostate cancer [18].
Regardless of the similarities between PSA and hK2, hK2 displays properties distinct from those of PSA. hK2 has been used in immunoassays to improve the accuracy of PCa screening $[17,20]$. In serum, most $\mathrm{hK} 2$ is in its free form, although the total levels of $h K 2$ are in the range of $1 \%$ total PSA but similar to that of fPSA [21]. As a tissue marker for $\mathrm{PCa}$, the hK2-specific immunostaining pattern differs from that of PSA, with an increased intensity in the $\mathrm{PCa}$ tumour and lymph node metastases compared to that observed in benign tissue $[22,23]$. Moreover, the very-low-to-no expression in other organs $[12,13]$ makes hK2 a potential target candidate for $\mathrm{PCa}$ imaging.

Owing to these potentially advantageous characteristics of hK2 as a PCa biomarker, we investigated the possibility of targeting free $\mathrm{hK} 2$ in an androgen-dependent $\mathrm{PCa}$ model using ${ }^{111}$ In-radiolabelled $11 \mathrm{~B} 6$ - a murine IgG $_{1}$ hK2-specific monoclonal antibody previously used in immunoassays for free hK2 [24]. Here, we discuss the results of in vivo hK2 imaging with ${ }^{111}$ In-labelled $11 \mathrm{~B} 6$ in ARpositive LNCaP xenografts.

\section{Methods}

\section{Antibody conjugation and radiolabelling}

The murine monoclonal antibody, 11B6, was first described and characterized by Vaisanen et al. [24] and was provided by the University of Turku (Turku, Finland) for this study. Conjugation and radiolabelling was performed as previously described by Tolmachev et al. [25]. Briefly, $2 \mathrm{mg}$ of $11 \mathrm{~B} 6$ was conjugated with the chelator CHX-A"-DTPA (B-355, Macrocyclics; Dallas, TX, USA) through the isothiocyanate functional group. A solution of $11 \mathrm{~B} 6$ (4 to $5 \mathrm{mg} / \mathrm{mL}$ in PBS) was adjusted to $\mathrm{pH} 9.2$ using $0.07 \mathrm{M}$ sodium borate buffer (Sigma Aldrich; St. Louis, MO, USA). CHXA"-DTPA was then added to the protein solution at a molar ratio of 3:1 (chelator to antibody) and incubated at $40^{\circ} \mathrm{C}$ with gentle shaking. The reaction was terminated after $4 \mathrm{~h}$, and CHX-A"-DTPA-11B6, henceforth referred to as DTPA-11B6, was separated from the free chelate by size-exclusion chromatography on a NAP-5 column (GE Healthcare; Uppsala, Sweden) equilibrated with $20 \mathrm{~mL}$ of $0.2 \mathrm{M}$ ammonium acetate buffer (Sigma Aldrich), $\mathrm{pH}$ 5.5. Conjugated $11 \mathrm{~B} 6$ was eluted with $1 \mathrm{~mL}$ of ammonium acetate buffer, and aliquoted samples were stored at $-20^{\circ} \mathrm{C}$.

For radiolabelling, approximately $125 \mu \mathrm{L}$ of DTPA-11B6 (approximately $1 \mu \mathrm{g} / \mu \mathrm{L}$ in $0.2 \mathrm{M}$ ammonium acetate buffer pH 5.5) was mixed with a predetermined amount (approximately 50 to $100 \mathrm{MBq}$ ) of ${ }^{111} \mathrm{InCl}_{3}$ (Mallinckrodt Medical; Dublin, Ireland), incubated at room temperature for 1.5 to $2 \mathrm{~h}$ and then purified on a NAP-5 column (GE Healthcare) equilibrated with PBS (Thermo Scientific; Waltham, MA, USA). Labelling efficiency and kinetics were monitored by instant thin-layer chromatography (ITLC) (Biodex, Shirley, 
NY, USA) eluted with $0.2 \mathrm{M}$ citric acid (Sigma Aldrich). In this system, the radiolabelled conjugate remains at the origin line, while free ${ }^{111}$ In and ${ }^{111}$ In-DTPA migrate with the solvent front. The radioactive distribution was determined using a Cyclone Storage Phosphor System with Optiquant quantification software (Perkin Elmer; Waltham, MA, USA).

\section{Binding kinetics with surface plasmon resonance}

The $11 \mathrm{~B} 6$ binding kinetics were analysed by surface plasmon resonance using a Biacore 2000 (Biacore AB; Uppsala, Sweden). The affinity of $11 \mathrm{~B} 6$ to hK2 before and after CHX-A"-DTPA conjugation was determined. The hK2 antigen, provided by the University of Turku (Department of Biotechnology; Turku, Finland), was produced and purified as previously described [26]. hK2 antigen $(25.9 \mu \mathrm{g} / \mathrm{mL}$ in $10 \mathrm{mM}$ sodium acetate buffer pH 4.0 (Sigma Aldrich)) was immobilized on a CM4 research grade chip (Biacore $\mathrm{AB}$ ) by amino coupling using N-hydroxysuccinimide (NHS), 1-ethyl-3-(3-dimethylaminopropyl) carbodiimide hydrochloride (EDC) and $1 \mathrm{M}$ ethanolamine hydrochloride- $\mathrm{NaOH}, \mathrm{pH} 8.5$, in a Biacore 2000 system. Samples were flown over two flow cells, one being a blank reference, in five different concentrations ranging from 0.5 to $100 \mathrm{nM}$ to detect eventual binding. One of the two flow cells contained immobilized hK2, while the other was served as a blank reference. The binding kinetics were studied in a 3-min-long association phase and a 15-min-long dissociation phase with a flow rate of $30 \mu \mathrm{L} / \mathrm{min}$, followed by regeneration with $25 \mathrm{mM}$ glycine buffer $\mathrm{pH}$ 2.7. Kinetic constants were calculated using a 1:1 Langmuir binding model with correction for mass transfer. BIAEvaluation 4.1 software (Biacore $A B$ ) was used for calculations.

\section{Stability studies}

The stability of ${ }^{111}$ In-DTPA-11B6 was assessed in triplicate by incubating the compound at $4^{\circ} \mathrm{C}$ in PBS buffer or at $37^{\circ} \mathrm{C}$ in murine serum collected from normal NMRI mice. For stability in PBS, $1 \mu \mathrm{L}(n=3)$ was taken at 1, 2, 3 and 7 days and analysed by ITLC. For stability in serum, $10 \mu \mathrm{L}$ of ${ }^{111}$ In-DTPA-11B6 (corresponding to $3 \mu \mathrm{g}$ of antibody with 0.8 to $0.9 \mathrm{MBq}{ }^{111} \mathrm{In}$ ) was mixed with $100 \mu \mathrm{L}$ of mouse serum. Approximately $20 \mu \mathrm{L}$ of each mixture was collected after 2, 3 and 9 days of incubation and analysed by SDS-PAGE on a NuPAGE $4 \%$ to $12 \%$ Bis-Tris gel (Invitrogen; Carlsbad, CA, USA) in MES buffer (200 V constant, approximately $30 \mathrm{~min}$ ). ${ }^{111}$ In-DTPA and free ${ }^{111}$ In diluted in PBS were run in parallel with the incubated sample as controls. The distribution of the samples along the gel was evaluated using a Cyclone Storage Phosphor System (Perkin Elmer).

\section{Cell lines}

LNCaP and DU145 were purchased from American Type Culture Collection (ATCC; Manassas, VA, USA) and cultured in RPMI 1640 medium (Thermo Scientific) supplemented with $10 \%$ foetal bovine serum (Thermo Scientific) with $100 \mathrm{U} / \mathrm{mL}$ penicillin and $100 \mu \mathrm{g} / \mathrm{mL}$ streptomycin (Thermo Scientific). The cells were maintained at $37^{\circ} \mathrm{C}$ in a humidified incubator at $5 \% \mathrm{CO}_{2}$ and were detached with trypsin-EDTA solution (Thermo Scientific).

\section{Animal models}

All animal experiments were conducted in compliance with the national legislation on laboratory animals' protection and with the approval of the Ethics Committee for Animal Research (Lund University, Sweden). Two animal models were used in this study, NMRI-Nu with subcutaneous (s.c.) xenografts and SCID mice with intra-tibial xenografts. NMRI-Nu mice (6-to-8-week-old, Taconic; Ry, Denmark) were inoculated in the right flank by s.c. injection of 5 to $8 \times 10^{6}$ cells in a $200 \mu \mathrm{L}$ of cell suspension of 1:1 mixture of medium with Matrigel (BD Biosciences; San Jose, CA, USA). Tumours were allowed to develop for 6 to 8 weeks. SCID mice (6-to-8-week-old male, Charles River; Charles River, NJ, USA) were maintained under isoflurane anaesthesia during surgery. For intra-tibial inoculations, the tibia was punctured using a 23 -gauge needle, and $1 \times 10^{5}$ LNCaP cells were injected into the tibial cavity. The puncture was closed with bone wax, the incision sutured and the animals received a palliative dose of Temgesic (Buprenorphine, RB Pharmaceuticals; Richmond, VA, USA) once daily for 3 days post-surgery. Intra-tibial tumours were allowed to develop for 8 to 10 weeks. Additionally, a group of normal NMRI mice $(n=4)$ were used to study the distribution of the tracer in healthy animals. Animals were euthanized by intraperitoneal (i.p.) injection with $20 \mu \mathrm{L}$ per gram of body weight KetalarRompun solution. (Ketalar, $10 \mathrm{mg} / \mathrm{mL}$; Pfizer; New York, NY, USA, and Rompun, $1 \mathrm{mg} / \mathrm{mL}$; Bayer Animal Health; Monheim, Germany).

\section{Biodistribution studies}

Biodistribution studies were conducted to evaluate the uptake of ${ }^{111}$ In-DTPA-11B6 in human prostate cancer LNCaP xenografts. Mice ( $n=3$ to 5 per time point) received ${ }^{111}$ In-DTPA-11B6 (0.4 to $0.6 \mathrm{MBq}, 20 \mu \mathrm{g}$ of mAb, in approximately $100 \mu \mathrm{L}$ of PBS) through intravenous (i.v.) tail vein injection. Blood and organs (including tumour) were taken at 4, 24, 48, 72 and $168 \mathrm{~h}$ post-injection, weighed and measured in a $\mathrm{NaI}(\mathrm{TI})$ well counter (Wallac Wizard 1480 Wizard, Perkin Elmer). The activity injected into each animal was measured and used to determine the count rate, in comparison with a standard solution of 
${ }^{111}$ In-DTPA-11B6. Data were corrected for background and physical decay.

Organ-specific uptake values were calculated as percent injected activity per gram of tissue $(\% \mathrm{IA} / \mathrm{g})$ or percent injected activity (\%IA). Among the organs resected were the lateral and ventral prostate, from now on referred to as prostate, and the submandibular glands, from now on called salivary glands.

\section{In vivo binding specificity}

In vivo competitive binding studies were performed to investigate the specificity of ${ }^{111}$ In-DTPA-11B6 to hK2. A 40-fold excess of non-labelled 11B6 was i.v. injected as a co-injection or at 168,120 and $48 \mathrm{~h}$ prior to an i.v. injection of ${ }^{111}$ In-DTPA-11B6 in hK2-positive LNCaP xenografts ( $n=3$ to 4 per pre-injection time point). Blood and organs (including tumour) were taken at $48 \mathrm{~h}$ postinjection of ${ }^{111}$ In-DTPA-11B6, weighed and analysed as above. The binding specificity was also evaluated by measuring the uptake of ${ }^{111}$ In-DTPA-11B6 in hK2negative DU145 xenografts expressing low levels of hK2 $(n=3)$ at $48 \mathrm{~h}$ post-injection, which are considered to be hK2-negative when compared to other PCa tumours.

\section{Small animal PET/SPECT/CT/MR imaging}

Animals were anaesthetized with $2 \%$ to $3 \%$ isoflurane gas (Baxter; Deerfield, IL, USA) for all imaging purposes. For SPECT/CT imaging, NMRI-nu mice with s.c. LNCaP xenografts (48 h post-injection, $n=4 ; 72 \mathrm{~h}$ post-injection, $n=3$; pre-dosed 11B6, $n=4$; co-injection 5A10, $n=3$ ) and SCID mice $(n=3)$ with intra-tibial LNCaP xenografts were i.v. injected with approximately $8 \mathrm{MBq}$ of ${ }^{111} \mathrm{In}$-DTPA-11B6 (approximately $20 \mu \mathrm{g}$ of $\mathrm{mAb}$ in $150 \mu \mathrm{L}$ of PBS) and imaged, for $1 \mathrm{~h}$, by using a preclinical SPECT/CT scanner (NanoSPECT/CT Plus, Bioscan; Washington, DC, USA) with the NSP-106 multi-pinhole mouse collimator. SPECT data were reconstructed using HiSPECT software (SciVis; Goettingen, Germany). CT imaging was done before each whole-body SPECT.

Pre-dosed mice were given $0.8 \mathrm{mg}$ of non-labelled $\mathrm{mAb}$ $48 \mathrm{~h}$ prior to injection of radiolabelled $\mathrm{mAb}$. Co-injections with ${ }^{111}$ In-DTPA-11B6 and $1.5 \mathrm{mg}$ of fPSA-specific 5A10 were done to evaluate the possible cross-reactivity of ${ }^{111}$ In-DTPA-11B6 with PSA.

The legs of the SCID mice were resected after imaging, and the radioactivity in the intra-tibial xenografted and the non-xenografted leg were measured in the $\mathrm{NaI}(\mathrm{Ti})$ well counter. Radiolabelling for SPECT of intra-tibial xenografts demonstrated $95 \%$ radiochemical purity and was injected directly without NAP-5 column purification. Verification of the intra-tibial tumour growth was performed by MR imaging. The legs were imaged in an $11.7 \mathrm{~T}(500 \mathrm{MHz}$ for protons) vertical bore MR camera (Agilent Technologies; Palo Alto, CA, USA) equipped with Varian 88/55 micro- imaging triple axis gradient coil $(1 \mathrm{~T} / \mathrm{m}$ maximum gradient strength). Samples were placed in the centre of a Millipede imaging probe (Agilent Technologies; Santa Clara, CA, USA), with an inner diameter of $40 \mathrm{~mm}$.

For PET/CT imaging, mice with LNCaP xenografts were i.v. injected with approximately $12 \mathrm{MBq}{ }^{18} \mathrm{~F}-\mathrm{FDG}$ $(n=4)$ or approximately $12 \mathrm{MBq}^{18} \mathrm{~F}$-Choline $(n=4)$ and imaged $1 \mathrm{~h}$ post-injection using a Bioscan NanoPET/CT Plus preclinical scanner for approximately $15 \mathrm{~min}$. Both SPECT/CT and PET/CT images were analysed using InVivoScope 2.0 software (inviCRO; Boston, MA, USA), and ROIs were drawn using the CT image as anatomical reference.

\section{Autoradiography and staining}

After SPECT imaging at 48 and 72 h, s.c. tumours were resected and embedded in Tissue-Tek ${ }^{\ominus}$ O.C.T $\mathrm{T}^{\mathrm{TM}}$ compound (Sakura Finetek; Alphen aan den Rijn, The Netherlands) and frozen on dry ice. The frozen samples were cryosectioned with a thickness of $20 \mu \mathrm{m}$ for autoradiography analysis on a Cyclone Storage Phosphor System. The tumour sections were stained with Mayer's hematoxylin and chromotrope 2R, Ch2R (both from Histolab; Gothenburg, Sweden), and scanned using a light-microscope slide scanner (Mirax Midi, Carl Zeiss; Oberkochen, Germany). Thresholds for the autoradiograms were set in Image J.1.47.

\section{Statistical analysis}

Data was analysed using the unpaired, two-tailed Student's $t$ test (Microsoft Excel or GraphPad Prism v.4). Differences at the $95 \%$ confidence level $(P<0.05)$ were considered to be statistically significant. Figures were produced with GraphPad Prism v.4 (GraphPad Software). All biodistribution data are shown as an average \%IA/g of 3 to 5 animals \pm SD (standard deviation) unless otherwise stated.

\section{Results}

\section{Radiolabelling and stability}

A radiolabelling yield of $58.6 \% \pm 12.5 \% \quad(n=3)$ was achieved. The radiochemical purity after NAP-5 purification was $>99 \%$ and for labelling with higher activities for imaging a specific activity of $0.30 \pm 0.03 \mathrm{MBq} / \mu \mathrm{g}(n=3)$. ${ }^{111}$ In-DTPA-11B6 was stable in PBS buffer at $4^{\circ} \mathrm{C}$ and in mouse serum at $37^{\circ} \mathrm{C}$. More than $95 \%$ of the radioactivity was still attached to the radioconjugate after 1 week in both conditions.

\section{Binding kinetics with surface plasmon resonance}

The binding kinetics of $11 \mathrm{~B} 6$ were analysed on a Biacore instrument. The affinity of 11B6 was high for hK2 and, although conjugation seemed to affect the association constant $\left(\mathrm{k}_{\mathrm{a}}\right)$ and the dissociation constant $\left(\mathrm{k}_{\mathrm{d}}\right)$, the calculated binding affinities $\left(K_{D}\right.$; calculated as $\left.k_{d} / k_{a}\right)$ were similar. 
The $K_{D}$ for $11 \mathrm{~B} 6$ and DTPA-11B6 to hK2 were estimated to be $(5.1 \pm 2.2) \times 10^{-11} \mathrm{M}$ and $6.6 \times 10^{-11} \pm 0.46 \times 10^{-11}$ $\mathrm{M}$, respectively. Representative sensorgrams of the $11 \mathrm{~B} 6$ and DTPA-11B6 binding kinetics are shown in the supplementary data (Additional file 1: Figure S1A,B).

\section{Biodistribution studies}

The biodistribution of ${ }^{111}$ In-DTPA-11B6 in NMRI mice with LNCaP xenografts revealed that the accumulation in tumour tissue increased threefold from $4 \mathrm{~h}(5.9 \pm 3.6 \% \mathrm{IA} / \mathrm{g})$ to a maximum at $48 \mathrm{~h}(19 \pm 0.78 \% \mathrm{IA} / \mathrm{g})$ (Figure 1a). Blood

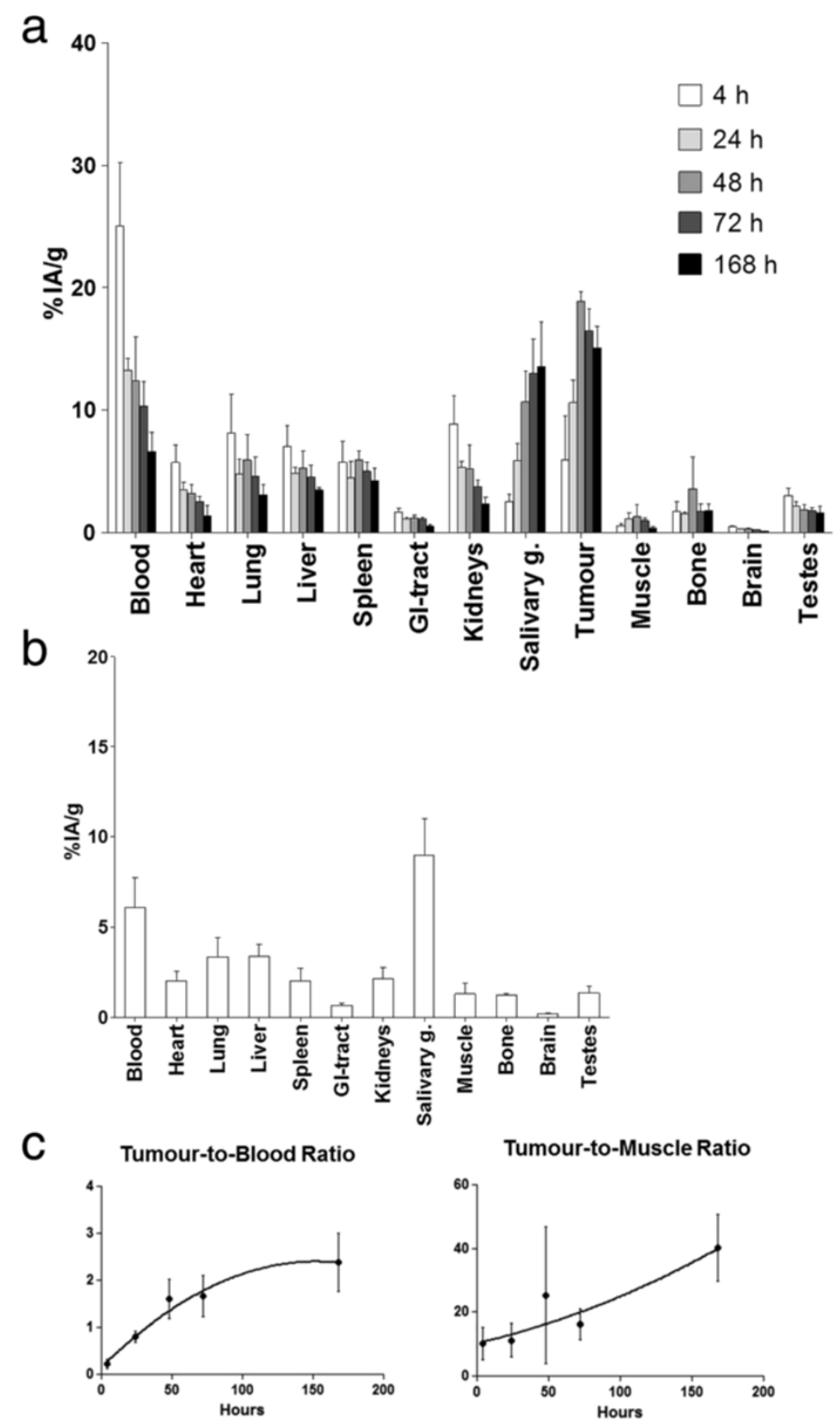

Figure 1 Biokinetics of ${ }^{111}$ In-DTPA-11B6. (a) Biodistribution of ${ }^{111}$ In-DTPA-11B6 in NRMI nude mice carrying LNCaP xenografts. \%IA/g \pm SD, 3 to 5 animals per time point. (b) Biodistribution of ${ }^{111}$ In-DTPA-11B6 in normal NMRI mice at $48 \mathrm{~h}$. An overall lower \%lA/g was observed as compared to tumour-bearing mice but with a retained high uptake in the salivary glands. (c) Left, tumour-to-blood ratio is steadily increasing over the time of the experiment. Right, tumour-to-muscle ratio. This high, increasing ratio explains the good visualization and contrast observed in SPECT/CT images. (See Figure 3) 
radioactivity was steadily cleared from the circulation over time, resulting in a tumour-to-blood ratio of 1.6 after 3 days and 2.4 at 1 week (Table 1). All organs showed constant, low uptake and low tissue-to-blood ratios $(\mathrm{Ti} / \mathrm{B})$, except for the salivary glands and highly vascularized organs, such as the spleen, liver and lung, suggesting no active antibody accumulation. Furthermore, the spleen, liver and lung showed steady increase in tumour-to-tissue $(\mathrm{T} / \mathrm{Ti})$ ratios. The $\mathrm{T} / \mathrm{Ti}$ ratios increased more than threefold between $4 \mathrm{~h}$ and 1 week (Table 2). The salivary gland accumulation followed a different pattern, more similar to that of the tumour, reaching a maximum at 1 week $(13.6 \pm 3.6 \% \mathrm{IA} / \mathrm{g})$. This elevated uptake could be due to cross-reactivity of ${ }^{111}$ In-DTPA-11B6 since most mice kallikreins are abundant in this organ [27], although mice are naturally deficient hK2 [16]. The high level of mice kallikreins in the salivary glands could be an explanation to retained salivary gland uptake even after pre-dosing (Figure $2 \mathrm{~b}$ ). A similar, but slightly lower, salivary gland uptake $(9.0$ $\pm 2.0 \% \mathrm{IA} / \mathrm{g}$ ) was seen in tumour-free NMRI mice at 48 $\mathrm{h}$ (Figure 1b). These mice exhibited a lower overall organ uptake of the tracer, which could be explained by the lack of shedded antigen circulating in the blood.

At 1 week, the steadily increasing $\mathrm{T} / \mathrm{Ti}$ ratios for blood and muscle were $2.4 \pm 0.62$ and $40 \pm 11$, respectively (Table 1, Table 2 and Figure 1c). Particularly high increases in $\mathrm{T} / \mathrm{Ti}$ were seen for the brain with a $\mathrm{T} / \mathrm{Ti}$ of $118 \pm 13$ at the end of the study. The high brain $\mathrm{T} / \mathrm{Ti}$ could probably be explained by the tight junctions of the epithelial cells of the brain vasculature and blood-brain barrier and then only reflecting the blood content.

Table 1 Tissue-to-blood (Ti/B) ratios of ${ }^{111}$ In-DTPA-11B6 in NMRI nude mice carrying LNCaP xenografts

\begin{tabular}{llllll}
\hline & $\mathbf{4 h}$ & $\mathbf{2 4} \mathbf{h}$ & $\mathbf{4 8} \mathbf{h}$ & $\mathbf{7 2} \mathbf{h}$ & $\mathbf{1 6 8} \mathbf{h}$ \\
\hline Tumour & $0.23 \pm 0.10$ & $0.80 \pm 0.12$ & $1.6 \pm 0.41$ & $1.7 \pm 0.44$ & $2.4 \pm 0.62$ \\
Heart & $0.23 \pm 0.02$ & $0.26 \pm 0.03$ & $0.26 \pm 0.03$ & $0.24 \pm 0.02$ & $0.22 \pm 0.16$ \\
Lung & $0.33 \pm 0.12$ & $0.36 \pm 0.07$ & $0.48 \pm 0.12$ & $0.43 \pm 0.07$ & $0.47 \pm 0.05$ \\
Liver & $0.28 \pm 0.04$ & $0.36 \pm 0.02$ & $0.43 \pm 0.03$ & $0.44 \pm 0.04$ & $0.55 \pm 0.14$ \\
Spleen & $0.23 \pm 0.06$ & $0.34 \pm 0.09$ & $0.50 \pm 0.09$ & $0.49 \pm 0.07$ & $0.64 \pm 0.06$ \\
Gl tract & $0.07 \pm 0.004$ & $0.08 \pm 0.003$ & $0.09 \pm 0.01$ & $0.11 \pm 0.04$ & $0.08 \pm 0.01$ \\
Kidneys & $0.35 \pm 0.05$ & $0.40 \pm 0.03$ & $0.41 \pm 0.05$ & $0.37 \pm 0.04$ & $0.35 \pm 0.03$ \\
Salivary & $0.10 \pm 0.01$ & $0.44 \pm 0.08$ & $0.86 \pm 0.11$ & $1.3 \pm 0.11$ & $2.1 \pm 0.38$ \\
Muscle & $0.02 \pm 0.003$ & $0.08 \pm 0.03$ & $0.09 \pm 0.05$ & $0.09 \pm 0.02$ & $0.06 \pm 0.01$ \\
Bone & $0.07 \pm 0.03$ & $0.12 \pm 0.01$ & $0.34 \pm 0.34$ & $0.17 \pm 0.05$ & $0.27 \pm 0.07$ \\
Brain & $0.02 \pm 0.003$ & $0.02 \pm 0.003$ & $0.02 \pm 0.003$ & $0.02 \pm 0.001$ & $0.02 \pm 0.003$ \\
Prostate & $0.06 \pm 0.01$ & $0.24 \pm 0.04$ & $0.14 \pm 0.04$ & $0.19 \pm 0.04$ & $0.20 \pm 0.05$ \\
Testes & $0.12 \pm 0.02$ & $0.16 \pm 0.02$ & $0.15 \pm 0.02$ & $0.17 \pm 0.02$ & $0.27 \pm 0.20$ \\
\hline & & & & &
\end{tabular}

Table 2 Tumour-to-tissue (T/Ti) ratios of ${ }^{111}$ In-DTPA-11B6 in NMRI nude mice carrying LNCaP xenografts

\begin{tabular}{llllll}
\hline & $\mathbf{4} \mathbf{h}$ & $\mathbf{2 4} \mathbf{h}$ & $\mathbf{4 8} \mathbf{h}$ & $\mathbf{7 2} \mathbf{~}$ & $\mathbf{1 6 8} \mathbf{h}$ \\
\hline Heart & $0.99 \pm 0.44$ & $3.0 \pm 0.13$ & $6.2 \pm 1.5$ & $6.7 \pm 1.7$ & $38 \pm 58$ \\
Lung & $0.75 \pm 0.43$ & $2.3 \pm 0.21$ & $3.5 \pm 1.4$ & $3.7 \pm 1.4$ & $5.2 \pm 1.5$ \\
Liver & $0.79 \pm 0.34$ & $2.2 \pm 0.44$ & $3.8 \pm 0.96$ & $3.5 \pm 1.0$ & $4.4 \pm 0.28$ \\
Spleen & $0.99 \pm 0.45$ & $2.5 \pm 0.46$ & $3.2 \pm 0.27$ & $3.1 \pm 0.57$ & $3.7 \pm 0.83$ \\
Gl tract & $3.5 \pm 1.6$ & $9.8 \pm 1.6$ & $17 \pm 3.5$ & $14 \pm 1.3$ & $30 \pm 8.2$ \\
Kidneys & $0.62 \pm 0.25$ & $2.0 \pm 0.24$ & $3.9 \pm 1.2$ & $4.2 \pm 0.71$ & $6.8 \pm 1.5$ \\
Salivary & $2.3 \pm 0.98$ & $1.9 \pm 0.24$ & $1.8 \pm 0.35$ & $1.2 \pm 0.32$ & $1.2 \pm 0.37$ \\
Muscle & $10 \pm 5$ & $11 \pm 5.3$ & $25 \pm 21$ & $16 \pm 4.8$ & $40 \pm 11$ \\
Bone & $3.5 \pm 0.81$ & $7.0 \pm 1.4$ & $7 \pm 3.9$ & $10 \pm 3.6$ & $9.2 \pm 2.6$ \\
Brain & $12 \pm 6.6$ & $39 \pm 4.5$ & $75 \pm 31$ & $74 \pm 22$ & $118 \pm 13$ \\
Prostate & $3.7 \pm 1.3$ & $3.5 \pm 0.99$ & $12 \pm 7.0$ & $8.2 \pm 3.0$ & $12 \pm 1.5$ \\
Testes & $1.9 \pm 0.82$ & $5.0 \pm 0.77$ & $11 \pm 2.2$ & $9.5 \pm 2.4$ & $11 \pm 3.9$ \\
\hline
\end{tabular}

In vivo binding specificity

Pre-injection with an excess of cold antibody $(0.8 \mathrm{mg}$ of cold $\mathrm{mAb}$ ) significantly decreased tumour accumulation of the labelled conjugate. The tumour accumulation was $7.2 \pm 0.53 \% \mathrm{IA} / \mathrm{g}$ at $48 \mathrm{~h}$ post-injection in mice that received a blocking dose of $11 \mathrm{~B} 6$ and was significantly lower to the $17 \pm 5.2 \% \mathrm{IA} / \mathrm{g}$ observed in non-blocked mice $(P=0.03)$ (Figure 2a). Furthermore, the DU145 hK2negative xenografts showed significantly lower $(P=0.01)$ tumour accumulation for ${ }^{111}$ In-DTPA-11B6, $4.8 \pm 0.86 \%$ $\mathrm{IA} / \mathrm{g}$ compared to $17 \pm 5.2 \% \mathrm{IA} / \mathrm{g}$ at $48 \mathrm{~h}$ (Figure $2 \mathrm{a}$ ).

The difference in specific uptake between tumour samples from pre-dosed mice $(n=16)$ and the reference group $(n=4)$ deteriorated with decreasing tumour weight, which skewed the tumour uptake data for the $120 \mathrm{~h}$ and co-injection time points (Figure $2 b$ ). For all groups, the specific uptake increased with tumour volume, but it was less in the pre-dosed groups (Figure 2c). This demonstrates that it is possible to block the uptake with cold 11B6 and remains effective over the span of 1 week. In other organs, no or small deviations from the non-blocked group were observed.

\section{Small animal PET/SPECT/CT/MR imaging}

Representative SPECT/CT images of LNCaP xenografts measured 2 to 3 days post-injection of ${ }^{111}$ In-DTPA-11B6 are shown in Figure 3a. The activity distribution in the SPECT images, with a high tumour-to-background contrast, is comparable to the high tumour-to-tissue ratios at these time points (Table 1). The high contrast was most likely attributed to the high affinity and specificity of the tracer.

The ROI analysis showed that the ratio of tumour to soft tissue of the contralateral leg was $6.1 \pm 1.5$ at $48 \mathrm{~h}(n=4)$ and $6.8 \pm 1.7$ at $72 \mathrm{~h}(n=3)$. ROI analysis also showed a tumour-to-heart ratio of $1.7 \pm 0.7$ and a tumour-to-liver 

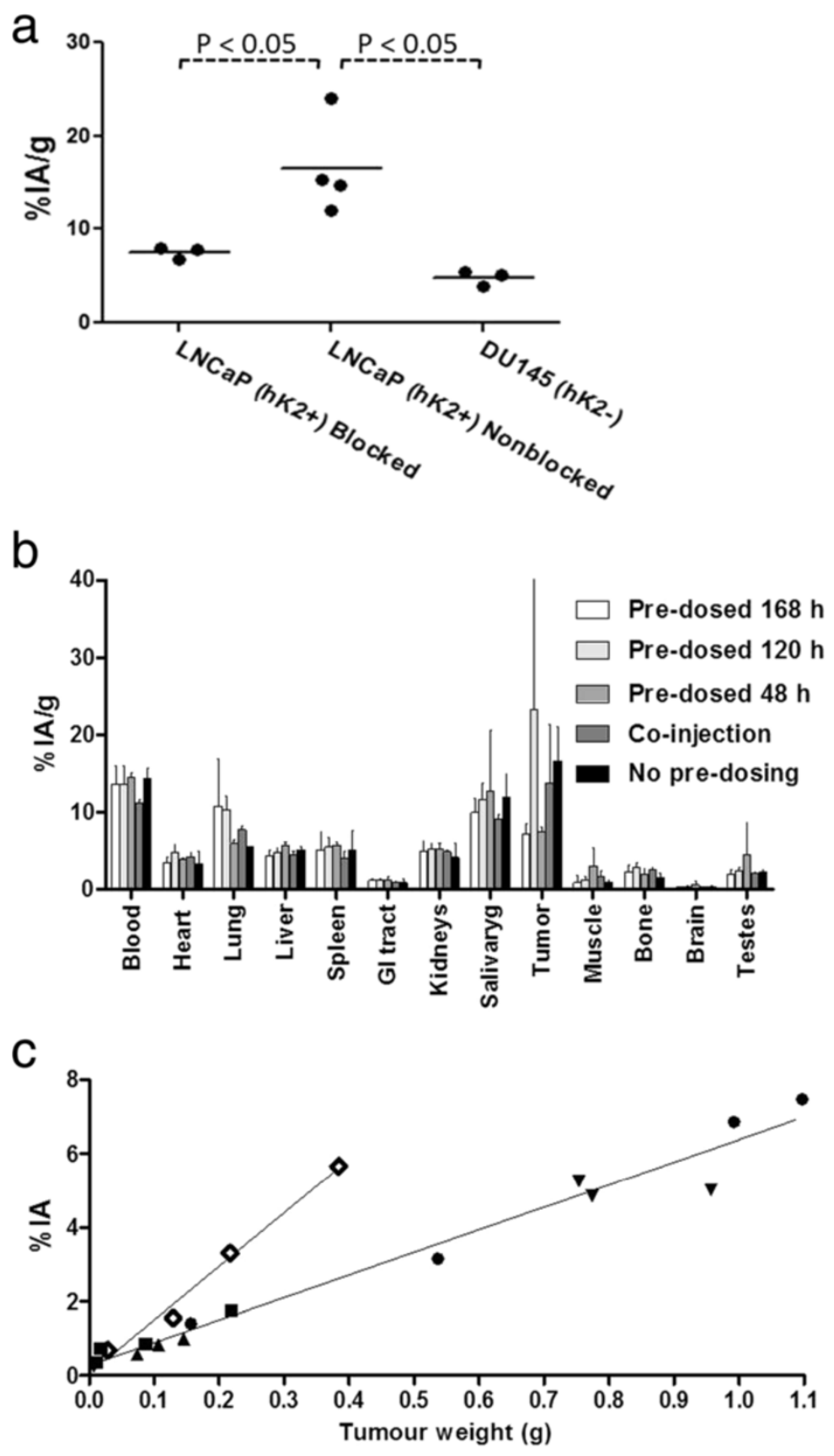

- Pre-dosed $168 \mathrm{~h}$. Pre-dosed $120 \mathrm{~h}$

- Pre-dosed $48 \mathrm{~h} \vee$ Co-injection

- No pre-dosing

Figure 2 In vivo binding specificity of ${ }^{111}$ In-DTPA-11B6 in NRMI nude mice. (a) Dot plot of tumour accumulation in blocked LNCaP, non-blocked LNCaP xenografts (hK2+) and DU145 (hK2-). There was a significant difference in active uptake between non-blocked LNCaP xenografts and the other groups. (b) Distribution of ${ }^{111}$ In-DTPA-11B6 $48 \mathrm{~h}$ post-injection in LNCaP xenografted NMRI mice, shown as \%IA/g \pm SEM with pre-dosing of $0.8 \mathrm{mg}$ of cold 11B6 antibody. (c) Dot plot with linear regression for pre-dosing and no pre-dosing as function of tumour weight. This shows that \% IA increases with tumour weight for both pre-dosed and normal uptake but that the uptake increases more in tumours with no pre-dosing. The slope calculated from linear regression was $6.1 \pm 0.27 \% \mathrm{IA} / \mathrm{g}$ for the pre-dosed groups and $14 \pm 1.2 \% \mathrm{IA} / \mathrm{g}$ with no pre-dosing. The $R^{2}$ value was 0.97 for the pre-dosed groups and 0.99 for xenografts without pre-dosing. As \% IA/g increases with smaller tumour volume this could explain the large difference seen in (b) between the different pre-dosing groups. By analysing \%IA instead, it seems that the effect of pre-dosing is retained over the studied time interval and that 11B6 in fact has a long tumour retention. 


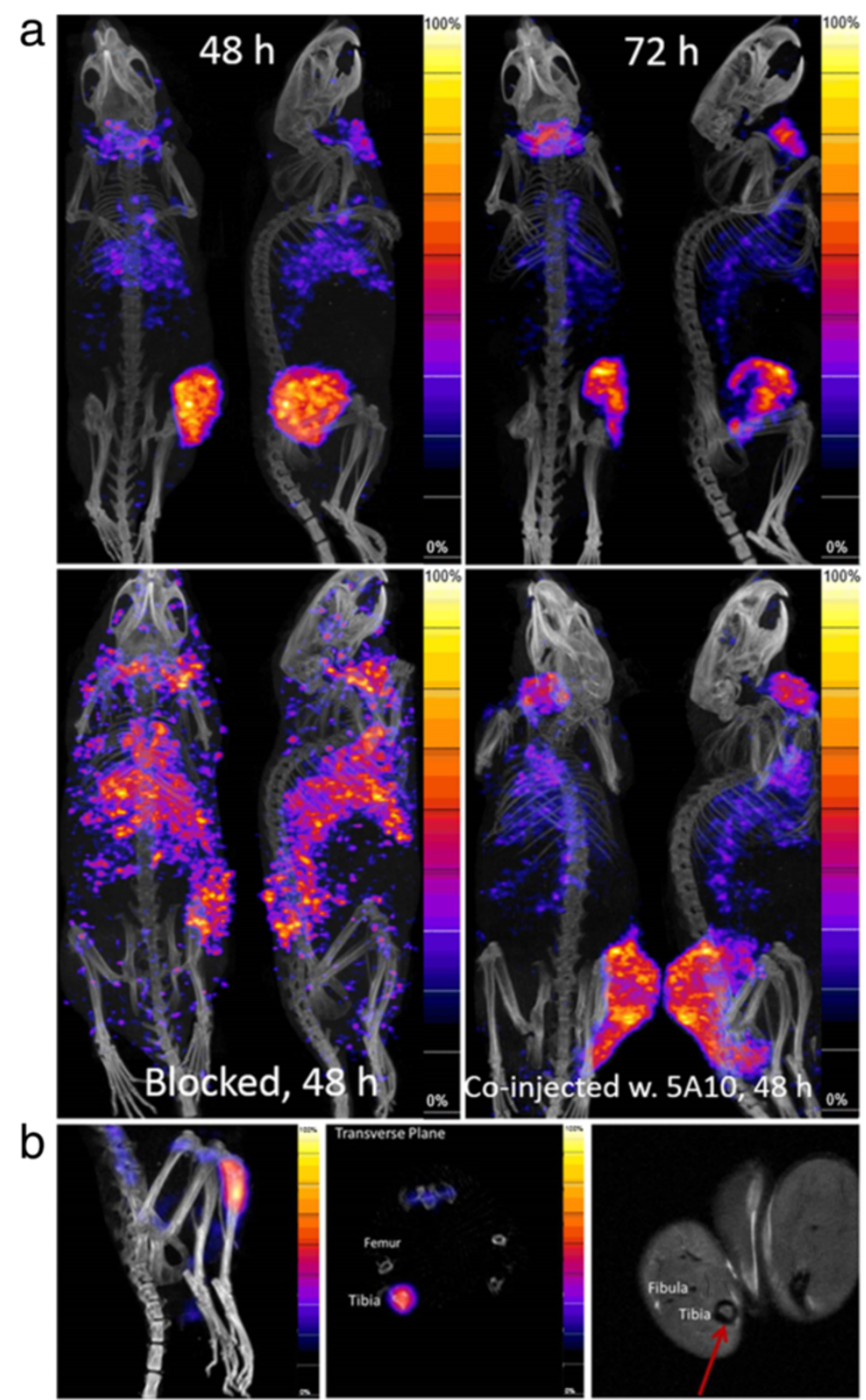

Figure 3 Preclinical SPECT/CT and MR images. (a) SPECT/CT images of four NMRI nude mice with LNCaP xenografts on the right flank. Top left, $48 \mathrm{~h}$ post-injection; Top right, $72 \mathrm{~h}$ post-injection; Bottom left, blocked mouse pre-injected with $0.8 \mathrm{mg}$ of cold antibody $48 \mathrm{~h}$ prior to injection of radiolabelled antibody. Bottom right, image of a mouse at $48 \mathrm{~h}$ co-injected with $1.5 \mathrm{mg}$ of unlabelled 5A10, which did not block the tumour uptake of ${ }^{111}$ In-DTPA-1 1B6. It should be noted that for the calculation of the T/B ratio (1.6) in the biodistribution and for the ROI analysis of the SPECT images with a derived T/heart ratio of 1.7, the whole tumour is taken into account. Since the colour scale in the SPECT image is scaled partially based upon high uptake areas of the tumour, a direct comparison between these (which are strikingly visible) and the T/B or T/heart (ROI) is not totally fair. (b) SPECT/MR images of SCID mice with intra-tibial xenograft in the left hind limb. Left, SPECT/CT side view, 48 h post-injection; middle, transverse SPECT/ $C T$ image slices of the same mouse. The accumulation of ${ }^{111}$ In-DTPA-11B6 in intra-tibial tumours is clearly shown in both images. Right, MR image of the same animal, where the growth of the intra-tibial tumour is well visualized (arrow).

ratio of $1.7 \pm 0.40$ at $48 \mathrm{~h}$ and $1.81 \pm 0.10$ and $1.9 \pm 0.04$, respectively, at $72 \mathrm{~h}$. This is similar to the tumour-to-tissue ratios derived from the biodistribution data. Interestingly, an obvious heterogeneous distribution of activity could be seen in the SPECT images of the tumours, later confirmed in autoradiograms (Figure 4a). High accumulation of activity was also detected in the salivary glands. For the blocked mice $(n=4)$, the ROI ratio at $48 \mathrm{~h}$ of the tumour to the 
a

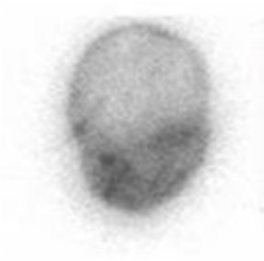

b

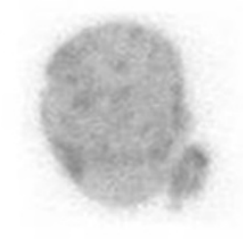

C
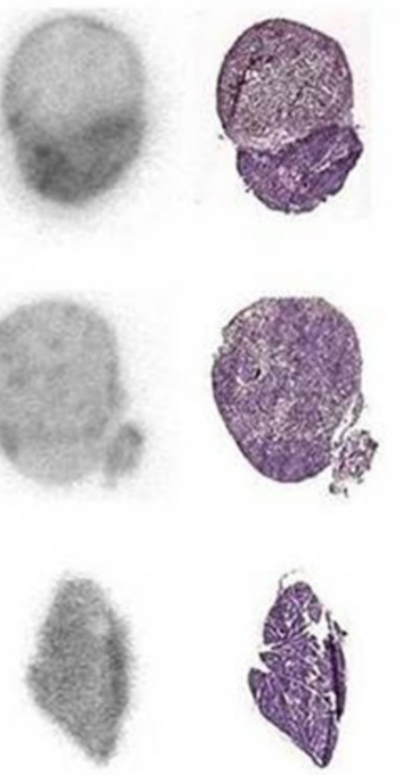
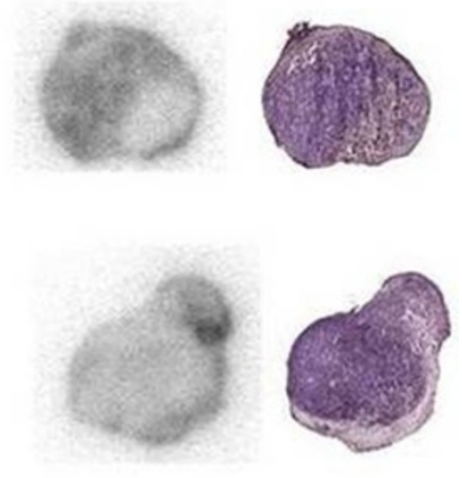

Figure 4 Autoradiograms and images of $\mathrm{H}$ - and Ch2R-stained tumour sections. (a) A heterogeneous distribution of activity with higher intensities in areas corresponding to a more intense level of staining with $\mathrm{H}$ and Ch2R, $48 \mathrm{~h}$ (left) and $72 \mathrm{~h}$ (right). (b) Stained sections and autoradiograms of two tumours resected at $48 \mathrm{~h}$ from mice pre-dosed with $0.8 \mathrm{mg}$ of cold $11 \mathrm{~B} 648 \mathrm{~h}$ prior to injection with ${ }^{111}$ In-DTPA-11B6. (c) An autoradiogram and stained section of a submandibular gland at $48 \mathrm{~h}$. This normal organ shows less heterogeneity in activity distribution and hematoxylin and Ch2R staining.

contralateral leg, heart and liver was $3.4 \pm 0.82,1.3 \pm$ 0.41 and $0.87 \pm 0.07$, respectively. The salivary gland uptake was not associated with detached indium-labelled CHX-A"-DTPA, as confirmed with SPECT/CT (Figure 5).

We also checked whether ${ }^{111}$ In-DTPA-11B6 could be used to detect PCa skeletal lesions. Osseous tumours were established in SCID mice through intra-tibial injections of LNCaP cells in the left hind limb, and SPECT/CT images were performed after i.v. injection of ${ }^{111}$ In-DTPA-11B6. The legs were resected and measured in the $\mathrm{NaI}(\mathrm{Ti})$ well counter, using the right nonxenografted legs as a negative control. The data showed an at least twofold uptake of ${ }^{111}$ In-DTPA-11B6 in the tibia compared to the reference leg, some of which might be due to inflammation induced by the surgical procedure. MR imaging confirmed tumour development within the bone (Figure $3 \mathrm{~b}$ ).

Although ${ }^{111}$ In-labelled 11 B6 showed a good stability in murine serum, we investigated whether the high uptake seen in the salivary glands could be a result of indium-labelled CHX-A"-DTPA, this was not confirmed (Figure 5, left). PET/CT images of NMRI-nude mice with s.c. LNCaP showed that ${ }^{18}$ F-FDG and ${ }^{18}$ F-Choline were hardly able to visualize the LNCaP xenografts, as compared to ${ }^{111}$ In-DTPA-11B6 (Figure 5, right). ROI analysis showed that the ratio of these xenografts to the soft tissue of the contralateral leg was $1.1 \pm 0.38$ for ${ }^{18} \mathrm{~F}$ FDG $(n=4)$ and $1.09 \pm 0.45$ for ${ }^{18} \mathrm{~F}$-Choline $(n=4)$.

\section{Autoradiography and staining}

Activity accumulation was confirmed to be heterogeneous in tumour sections at both 48 and $72 \mathrm{~h}$. Comparative autoradiograms between the pre-dosed (Figure 4b) and nonpre-dosed mice (Figure 4a) showed that for the former, activity was confined to areas with high hematoxylin and Ch2R staining, i.e. areas of high cell density, whereas the activity in tumours from pre-dosed animals was found in areas with low hematoxylin and Ch2R staining. This difference could be due to an active uptake in the viable regions, blocked in the latter by pre-dosing with cold antibody. The activity distribution was more homogenous in the salivary glands of non-pre-dosed mice, indicating an uptake in all glandular structures in this organ (Figure 4c).

\section{Discussion}

There is a clinical need for improved methods to detect and stage prostate cancer. The imaging of androgenregulated prostate-specific antigens overexpressed in prostate cancer, such as PSA, would be advantageous for diagnosing and monitoring the disease. In this study, we demonstrated the ability for a novel radiotracer targeting free $\mathrm{hK} 2$, a prostate-specific kallikrein homologous to PSA, to specifically target and image hK2 expression in AR- and hK2-positive LNCaP xenografts. Significantly, ${ }^{111}$ In-DTPA-11B6 exhibited strong targeting in both subcutaneous and intra-tibial bone xenografts, with a 


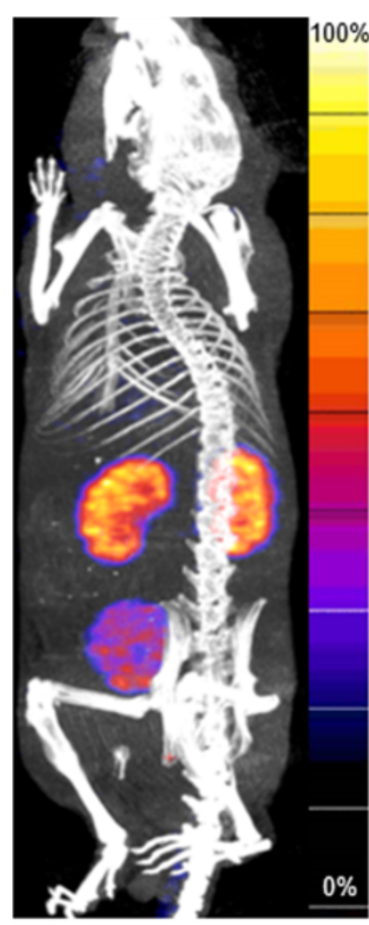

${ }^{111}$ In-CHX-A"-DTPA

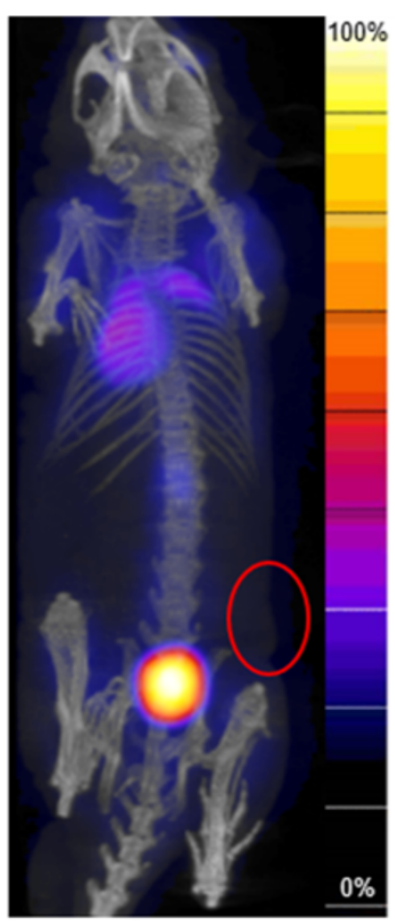

${ }^{18} \mathrm{~F}-\mathrm{FDG}$

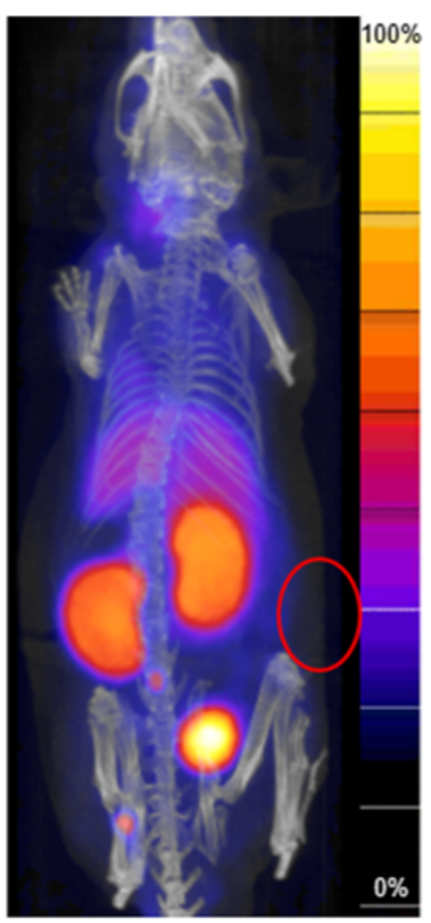

${ }^{18} \mathrm{~F}-\mathrm{FCH}$

Figure $5{ }^{111} \mathrm{In}-\mathrm{CHX}-\mathrm{A}$ "-DTPA SPECT/CT and ${ }^{18}$ F-FDG and ${ }^{18}$ F-Choline PET/CT images. SPECT/CT images (left) of non-tumour bearing NMRI nude mice showing localization of ${ }^{111}$ In-CHX-A"-DTPA in the kidneys (0.5 h post-injection). PET/CT images of s.c. LNCaP xenografts $1 \mathrm{~h}$ after injection with ${ }^{18} \mathrm{~F}$-FDG and ${ }^{18} \mathrm{~F}$-Choline, respectively (middle and right). Tumours, indicated by red circles, were not well visualized with these radiotracers.

mean tumour uptake of $19 \% \mathrm{IA} / \mathrm{g}$ at $48 \mathrm{~h}$ in subcutaneous tumours (Figure 1a). The elevated uptake of ${ }^{111} \mathrm{In}$ DTPA-11B6 in the salivary glands was an unexpected result of this study; however, several glandular kallikreins are synthesized by the salivary glands in mice [27]. Since we see an uptake in the salivary glands of both NMRI nude and normal non-xenografted NMRI mice, it seems likely that ${ }^{111}$ In-DTPA-11B6 cross-reacts with kallikreins expressed in the salivary glands of these mice.

The tumour accumulation of ${ }^{111}$ In-DTPA-11B6 was hK2-specific, as verified by the low accumulation in the negative control, DU145 xenografts and by competitive binding assays using excess cold non-labelled antibody. Furthermore, tumour accumulation slowly decreased after day 2 but was not as rapid as blood clearance at the same time points. This gave a steadily increasing pattern in tumour-to-blood $(\mathrm{T} / \mathrm{B})$ ratios over time, from $1.6 \pm 0.41$ at $48 \mathrm{~h}$ to $2.4 \pm 0.62$ at 1 week (Table 1 ) Though the blood clearance of ${ }^{111} \mathrm{In}-11 \mathrm{~B} 6$ is comparable to other full-sized IgGs, the high tumour uptake, an increasing $\mathrm{T} / \mathrm{B}$ ratio and a comparatively low liver uptake, implicates the usefulness of this radiotracer for imaging. Further, most tissues seem to display blood kinetics, except for a faint active uptake by the liver, spleen and lung compared to the high accumulation rate observed in the tumour and salivary gland tissue. Tissues such as the muscle and brain gave high $\mathrm{T} / \mathrm{Ti}$ ratios of $40 \pm 11$ and $118 \pm 13$, respectively, at 1 week, whereas the liver and spleen have lower $\mathrm{T} / \mathrm{Ti}$ ratios of $4.4 \pm 0.28$ and $3.7 \pm$ 0.83 , respectively. There could be some concerns raised about ${ }^{111}$ In-DTPA-11B6 binding to free hK2 in the blood. However, the blocking with cold non-labelled antibody does not seem to change the amount of the blood activity significantly at $48 \mathrm{~h}$ post-injection. Also, in the control group with DU145 xenografts, there was no significant difference in blood activity at $48 \mathrm{~h}$, but a significant difference in tumour uptake was found. One interesting observation from the pre-dosing studies was that the \%IA as a function of the tumour size (Figure 2c) differed between the pre-dosed (all pre-dosed/co-injected groups) and non-blocked tumours if these were not too small in tumour size. This explains the large deviation for the pre-dosing at $120 \mathrm{~h}$ in Figure $2 \mathrm{~b}$. These results also suggest a long tumour retention of the antibody, something also seen in the $\mathrm{T} / \mathrm{B}$ ratio.

Despite the high sequence homology between PSA and hK2, the 11B6 antibody did not show any crossreactivity to PSA. This was supported by the fact that co-injection with a high dose of PSA-specific 5A10 antibody did not hinder the uptake of ${ }^{111}$ In-DTPA-11B6 in LNCaP xenografts (Figure 3a). This highlights the possibility of concurrent use. As both $K L K 3$ and $K L K 2$ genes 
are regulated by AR signalling, it is reasonable to theorize that the 11B6 antibody could be used to image AR signalling in prostate cancer in a similar fashion to that reported with $5 \mathrm{~A} 10$ [18].

In addition, the tracer's specificity was further confirmed by the distinct differences seen between pre-dosed and non-pre-dosed LNCaP xenografts. This difference was also validated by both SPECT and in excised xenografts (Figure 3a, Figure 2). Furthermore, autoradiograms demonstrate that the localization of the labelled antibody to a dense PCa tissue could be changed or blocked by pre-dosing (Figure 4a,b). Although the underlying physiology remains unknown, this could be due to a decrease in an active uptake and/or the blocking of a pool of free hK2 in tumour tissue.

Based on our data, we believe that radiolabelled monoclonal antibodies such as ${ }^{111}$ In-DTPA-11B6 have an immense value for use in the imaging, staging and evaluation of advanced $\mathrm{PCa}$. The favourable $\mathrm{T} / \mathrm{Ti}$ ratios also suggest that 11B6 could be used as a moiety for delivering high absorbed doses in radioimmunotherapy, whereas ${ }^{111} \mathrm{In}$ radiolabelled 11B6 could potentially be used for patientindividualized dose planning.

\section{Conclusions}

${ }^{111}$ In-DTPA-11B6 is a new radiotracer for SPECT/CT imaging of hK2-expressing prostate cancer. To our knowledge, this is the first study using $h K 2$ as a target for immune imaging. The favourable biokinetics, high tumour accumulation and low normal organ uptake observed with ${ }^{111}$ In-DTPA-11B6 underscore its potential as a novel PCa imaging agent for the detection of metastatic PCa and for monitoring anti-androgen therapy. Because of its immunogenicity, the murine form of 11B6 is not suitable for future clinical trials. Nevertheless, the therapeutic potential of $11 \mathrm{~B} 6$ in radioimmunotherapy applications is under investigation, as well as a humanised version of 11B6, which is currently in development.

\section{Additional file}

Additional file 1: Figure S1. Surface plasmon resonance. The binding kinetics of 11B6 and DTPA-11B6 analysed by a Biacore 2000 Sensorgram of A. $11 \mathrm{~B} 6$ to hK2, before conjugation and B. DTPA-11B6 to hK2, after conjugation. $\mathrm{ka}, \mathrm{kd}$ and $\mathrm{KD}$ for $11 \mathrm{~B} 6$ and DTPA-11B6 are displayed below the graphs.

\section{Competing interests}

DU, SES and HL are shareholders of DiaProst (Lund, Sweden) who holds a patent for hK2 targeting. DU is the inventor of hK2 targeting (US Patent No. 20110097276). HL holds patents for free PSA, hK2 and intact PSA assays. TT holds stock options in DiaProst. DiaProst has not financed any part of the work conducted in this study.

\section{Authors' contributions}

OVT and TT were responsible for the study design, took part in carrying out all parts of the study, analysis and drafted the manuscript. DU contributed to the conception of the study, took part in carrying out the intra-tibial study and aided in manuscript revision. SES participated in the study conception, design, analysis and drafting of the manuscript. SEA and $A B$ participated in the biodistribution study, the in vivo binding specificity study and manuscript revisions. KP contributed to the study conception, production of $11 \mathrm{~B} 6 \mathrm{mAb}$ and manuscript revisions. HL contributed to the conception of the study and manuscript revisions. All authors read and approved the final manuscript.

\section{Acknowledgements}

We wish to thank Anna Åkesson and Susanne Strömblad at the Department of Medical Radiation Physics and Gustav Grafström and Adnan Bibic at Lund University Bioimaging Center (LBIC) for technical assistance. LBIC, Lund University is gratefully acknowledged for providing experimental resources. We give special thanks to Bo Jansson, Magdalena Godzwon and Anne Ljungars at Bioinvent for help with the Biacore analysis. OVT was supported by The Research School in Pharmaceutical Sciences (FLÄK, Lund University) and DU by the David $\mathrm{H}$. Koch Young Investigator Award from the Prostate Cancer Foundation. In addition, this study was performed with generous support from the Swedish Cancer Foundation, the Swedish Science Council, Mrs. Berta Kamprad's Foundation, Gunnar Nilsson's Foundation, Percy Falk's Foundation, government funding of clinical research within the NHS (National Health Service) Lund University, Sweden (ALF), the National Cancer Institute (R01CA160816 and P50-CA92629), the Sidney Kimmel Center for Prostate and Urologic Cancers, the National Institute for Health Research (NIHR) Oxford Biomedical Research Centre Program, Swedish Cancer Society (project no. 11-0624) and Fundación Federico SA.

\section{Author details}

1Department of Medical Radiation Physics, Lund University, Barngatan 2:1, S-211 85 Lund, Sweden. ${ }^{2}$ Department of Surgery (Urology), Memorial Sloan-Kettering Cancer Center, 1275 York Avenue, New York, NY 10065, USA. ${ }^{3}$ Department of Clinical Sciences, Div. Urological Cancers, Lund University, Jan Waldenstroms gata 5, S-205 02 Malmö, Sweden. ${ }^{4}$ Department of Biochemistry and Food Chemistry/Biotechnology, University of Turku, Tykistökatu 6A BioCity, 20520 Turku 52, Turku, Finland. ${ }^{5}$ Department of Laboratory Medicine and Medicine (GU Oncology), Memorial Sloan-Kettering Cancer Center, 1250 York Avenue, New York, NY 10065, USA. ${ }^{6}$ Nuffield Department of Surgical Sciences, University of Oxford, Oxford, UK. ${ }^{7}$ Department of Laboratory Medicine, University Hospital UMAS, Lund University, Malmö, Sweden. ${ }^{8}$ Lund University Bioimaging Center, BMC, Lund University, Klinikgatan 32, S-222 42 Lund, Sweden.

Received: 5 June 2014 Accepted: 7 September 2014

Published online: 19 September 2014

\section{References}

1. Crawford ED: Epidemiology of prostate cancer. Urology 2003, 62:3-12.

2. Welch HG, Black WC: Overdiagnosis in cancer. J Natl Cancer Inst 2010, 102:605-613.

3. Schroder FH, Hugosson J, Roobol MJ, Tammela TL, Ciatto S, Nelen V, Kwiatkowski M, Lujan M, Lilja H, Zappa M, Denis LJ, Recker F, Berenguer A, Määttänen L, Bangma CH, Aus G, Villers A, Rebillard X, van der Kwast T, Blijenberg B, Moss $\mathrm{SM}$, De Koning $\mathrm{HJ}$, Auvinen A: Screening and prostate-cancer mortality in a randomized European study. New Engl J Med 2009, 360:1320-1328.

4. Singh G, Lakkis CL, Laucirica R, Epner DE: Regulation of prostate cancer cell division by glucose. J Cell Physiol 1999, 180:431-438.

5. Pinski J, Parikh A, Bova GS, Isaacs JT: Therapeutic implications of enhanced $\mathrm{G}(0) / \mathrm{G}(1)$ checkpoint control induced by coculture of prostate cancer cells with osteoblasts. Cancer Res 2001, 61:6372-6376.

6. Souvatzoglou M, Weirich G, Schwarzenboeck S, Maurer T, Schuster T, Bundschuh RA, Eiber M, Herrmann K, Kuebler H, Wester HJ, Hoefler H, Gschwend J, Schwaiger M, Treiber U, Krause BJ: The sensitivity of [11C] choline PET/CT to localize prostate cancer depends on the tumor configuration. Clin Cancer Res 2011, 17:3751-3759.

7. Culig Z, Klocker H, Bartsch G, Hobisch A: Androgen receptors in prostate cancer. Endocr Relat Cancer 2002, 9:155-170.

8. Larson SM, Morris M, Gunther I, Beattie B, Humm JL, Akhurst TA, Finn RD, Erdi Y, Pentlow K, Dyke J, Squire O, Bornmann W, McCarthy T, Welch M, Scher H: Tumor localization of 16beta-18 F-fluoro-5alpha-dihydrotestosterone versus $18 \mathrm{~F}-\mathrm{FDG}$ in patients with progressive, metastatic prostate cancer. J Nucl Med 2004, 45:366-373. 
9. Haseman MK, Rosenthal SA, Polascik TJ: Capromab Pendetide imaging of prostate cancer. Cancer Biother Radio 2000, 15:131-140.

10. Evans MJ, Smith-Jones PM, Wongvipat J, Navarro V, Kim S, Bander NH, Larson SM, Sawyers CL: Noninvasive measurement of androgen receptor signaling with a positron-emitting radiopharmaceutical that targets prostatespecific membrane antigen. Proc Natl Acad Sci U S A 2011, 108:9578-9582.

11. Luthi-Carter R, Barczak AK, Speno H, Coyle JT: Molecular characterization of human brain $\mathrm{N}$-acetylated alpha-linked acidic dipeptidase (NAALADase). J Pharmacol Exp Ther 1998, 286:1020-1025.

12. Olsson AY, Bjartell A, Lilja $H$, Lundwall A: Expression of prostate-specific antigen (PSA) and human glandular kallikrein 2 (hK2) in ileum and other extraprostatic tissues. Int J Cancer 2005, 113:290-297.

13. Thorek DL, Evans MJ, Carlsson SV, Ulmert D, Lilja H: Prostate-specific kallikrein-related peptidases and their relation to prostate cancer biology and detection. Established relevance and emerging roles. Thromb Haemost 2013, 110:484-492.

14. Young CY, Andrews PE, Montgomery BT, Tindall DJ: Tissue-specific and hormonal regulation of human prostate-specific glandular kallikrein. Biochemistry 1992, 31:818-824.

15. Schedlich $\amalg$, Bennetts $B H$, Morris BJ: Primary structure of a human glandular kallikrein gene. DNA 1987, 6:429-437.

16. Lawrence MG, Lai J, Clements JA: Kallikreins on steroids: structure, function, and hormonal regulation of prostate-specific antigen and the extended kallikrein locus. Endocr Rev 2010, 31:407-446.

17. Rittenhouse $H G$, Finlay JA, Mikolajczyk SD, Partin AW: Human kallikrein 2 (hK2) and prostate-specific antigen (PSA): two closely related, but distinct, kallikreins in the prostate. Crit Rev Clin Lab Sci 1998, 35:275-368.

18. Ulmert D, Evans MJ, Holland JP, Rice SL, Wongvipat J, Pettersson K, Abrahamsson PA, Scardino PT, Larson SM, Lilja H, Lewis JS, Sawyers CL: Imaging androgen receptor signaling with a radiotracer targeting free prostate-specific antigen. Cancer Discov 2012, 2:320-327.

19. Evans-Axelsson S, Ulmert D, Orbom A, Peterson P, Nilsson O, Wennerberg J, Strand J, Wingardh K, Olsson T, Hagman Z, Tolmachev V, Bjartell A, Lilja H, Strand SE: Targeting free prostate-specific antigen for in vivo imaging of prostate cancer using a monoclonal antibody specific for unique epitopes accessible on free prostate-specific antigen alone. Cancer Biother Radiopharm 2012, 27:243-251.

20. Stephan C, Jung K, Lein M, Diamandis EP: PSA and other tissue kallikreins for prostate cancer detection. Eur J Cancer 2007, 43:1918-1926.

21. Steuber T, Vickers AJ, Serio AM, Vaisanen V, Haese A, Pettersson K, Eastham JA Scardino PT, Huland H, Lilja H: Comparison of free and total forms of serum human kallikrein 2 and prostate-specific antigen for prediction of locally advanced and recurrent prostate cancer. Clin Chem 2007, 53:233-240.

22. Darson MF, Pacelli A, Roche P, Rittenhouse HG, Wolfert RL, Saeid MS, Young CY, Klee GG, Tindall DJ, Bostwick DG: Human glandular kallikrein 2 expression in prostate adenocarcinoma and lymph node metastases. Urology 1999, 53:939-944.

23. Darson MF, Pacelli A, Roche P, Rittenhouse HG, Wolfert RL, Young CY, Klee GG, Tindall DJ, Bostwick DG: Human glandular kallikrein 2 (hK2) expression in prostatic intraepithelial neoplasia and adenocarcinoma: a novel prostate cancer marker. Urology 1997, 49:857-862.

24. Vaisanen V, Eriksson S, Ivaska KK, Lilja H, Nurmi M, Pettersson K: Development of sensitive immunoassays for free and total human glandular kallikrein 2. Clin Chem 2004, 50:1607-1617.

25. Tolmachev $\mathrm{V}$, Wallberg $\mathrm{H}$, Andersson $\mathrm{K}$, Wennborg A, Lundqvist $\mathrm{H}$, Orlova $\mathrm{A}$ : The influence of Bz-DOTA and CHX-A"-DTPA on the biodistribution of ABD-fused anti-HER2 affibody molecules: implications for (114 m)lnmediated targeting therapy. Eur J Nucl Med 2009, 36:1460-1468.

26. Lovgren J, Tian S, Lundwall A, Karp M, Lilja H: Production and activation of recombinant hK2 with propeptide mutations resulting in high expression levels. Eur J Biochem 1999, 266:1050-1055.

27. Yousef GM, Diamandis EP: The new human tissue kallikrein gene family: structure, function, and association to disease. Endocr Rev 2001, 22:184-204.

doi:10.1186/s13550-014-0051-5

Cite this article as: Vilhelmsson Timmermand et al:: Preclinical imaging of kallikrein-related peptidase 2 (hK2) in prostate cancer with a ${ }^{111} \mathrm{In}$ radiolabelled monoclonal antibody, 11B6. EJNMMI Research 2014 4:51.

\section{Submit your manuscript to a SpringerOpen ${ }^{\odot}$ journal and benefit from:}

- Convenient online submission

$\checkmark$ Rigorous peer review

- Immediate publication on acceptance

- Open access: articles freely available online

- High visibility within the field

- Retaining the copyright to your article

Submit your next manuscript at $\gg$ springeropen.com 